\title{
The Pathophysiology of Virulence of the COVID-19
}

Shazia Tabassum Hakim ${ }^{1}$, Frederick Tilghman Boyd ${ }^{1}$, Joseph Angel de Soto ${ }^{1}$

${ }^{1}$ School of Science, Technology, Engineering and Math, Dine College, Tsaile AZ 86566

Corresponding Author:

Joseph Angel de Soto

1 Circle Dr Route 64, Tsaile AZ 86566

Angeldesoto63@gmail.com 


\section{Abstract}

Background: On Dec 19, 2019, the public health department of China reported that an outbreak of pneumonia was caused by a novel Coronavirus. The virulence of the new virus COVID-19 was much greater than either the SARs and MERSs viruses and on March 11, 2020, the World Health Department (WHO) declared a worldwide pandemic. Understanding the pathophysiology of virulence of the SARS-COV-2 virus is absolutely necessary for understanding the transmission, virulence factors, reduce risk factors, clinical presentation, predict outcomes of the disease and provide guidance for any current or future treatment protocols. Methodology: A comprehensive PubMed search was performed during December 20, 2019 and April 03, 2020, utilizing the words: Wuhan Virus, COVID-19, SARs coronavirus, ACE2, S-protein, virulence, clinical presentation, epidemiology, genome, treatment, structure, MERs, pathogenesis and/or pathology alone and in combination with other terms. Each paper was evaluated by three content experts for quality, reproducibility, credibility and reputation of the journal. Results: The SARSCOV-2 virus is much more virulent than either the SAR's or MER's virus and its ability to cause serious disease inversely corresponds to the person's ability to produce T-cells which declines linearly with age. The ACE2 receptor binding site does not vary among different ethnic groups but do in ACE-2 expression levels. This variance in expression level may explain for different infectivity rates among men and women and predict and explain different susceptibilities to infection by different ethnic groups. Furthermore, by understanding the underlying pathophysiology one can explain and provide guidance to the clinical effectiveness of any treatment. Conclusions: The underlying pathophysiology of COVID-19 explains not only the virulence, and clinical presentation, but, explains at a molecular level the comorbidity risk factors such as hypertension, sex, and age. Ethnic and anatomic expression patterns of ACE-2 
and associated pathophysiology suggests that Native Americans and Asians may be particularly susceptible to this disease.

\section{Introduction}

A mysterious illness started from Wuhan, China, has sickened millions throughout the world. It was late November 2019 when patients with pneumonia like symptoms started to come and soon on December 19, 2019 the first case of a novel Coronavirus patient was reported officially by public health department of China. Chinese scientists discovered a new strain of coronavirus that is apparently and genetically different from previously known strains of famous coronaviruses like Sever Acute Respiratory Syndrome (SARS) and Middle East Respiratory Syndrome (MERS) coronavirus (CoV). This is a newly emerged strain that is currently testing the capabilities of this modern world for dealing with unfamiliar pathogens like this Novel Coronavirus SARS-2 (SARS-CoV-2) which causes COVID-19. As per briefing of Wuhan's Municipal Health and Health Commission on December 31, 2020, 27 cases were reported with similar clinical manifestations of fever and difficulty in breathing and their chest radiographs showed bilateral lung infiltrative lesions. Overall, the relentlessness of this COVID-19 is unquestionable. Because of the exponential growth of infection, on March 11, 2020; WHO declared this COVID-19 a pandemic. As of today March 31 $1^{\text {st }}, 2020$ with spread through 177 countries of the world, the COVID-19 remains severe, continuously expanding with worldwide 803,650 cases and biggest number of cases have been reported from US (164,785), Italy, $(101,739)$ Spain $(94,417)$, and China (82,276) (Johns Hopkins COVID data accessed on March $31^{\text {st }}$ ). In clinical practice, COVID-19 patients are still mainly affected by the severe respiratory system infection, but evidence of damage to other system organs such as cardiovascular 
complications has also been reported (Yao et al, 2020; Hu, Wang, \& Zhu 2020; Madjid et al., 2020).

The virus is highly contagious, case fatality rate for COVID-19 varies from place to place and country to country with current reported cases growing exponentially. Spreading quickly, affecting mainly elderly and populations with multiple underlying disorders but cases and deaths in young adults have also been reported including one death reported from Illinois of a patient under the age of 1 year. Global trends indicate that SARS-CoV-2 may reach $30-40 \%$ of the population, some of the communities may wiped out, and its spread will not slow down without accelerated human involvement in the near future in practices including social distancing, diagnostics, treatment and vaccination (Hagen, 2020). Exacerbating this is the fact the virus can spread from asymptomatic carriers (Rothe et al., 2020)

Normally flu, common cold and influenza are the infections that we observe during winter and spring, so is viral pneumonia. Viruses that cause viral pneumonia are commonly influenza/ orthomyxo viruses, others include parainfluenza viruses, adenoviruses, rhinoviruses, cytomegaloviruses, and coronaviruses. These infections might also result in spread or outbreak in small communities or regions, with most common clinical manifestations of fever, soreness, dyspnea, and lung infiltration. Complications are mainly associated with virus load, virulence, route of infection, age and immune status of the host. Higher SARS-CoV-2 viral loads might worsen outcomes, and data from China suggests the viral load is higher in patients with more severe disease. The amount of virus exposure at the start of infection - the infectious dose - may increase the severity of the illness and is also linked to a higher viral load (Heneghan et al., 2020). Majority of the cases are manageable with complete bed rest supplemented with supportive therapies. 
Coronaviruses (CoVs) are named for the crown-like spikes on their surface and belong to the family Coronaviridae within the order Nidovirales. Members of this large family of viruses can cause respiratory, enteric, hepatic, and neurological diseases in broad group of animals including humans, camels, cattle, cats, bats, birds, snakes, mice and other wild animals. Since the mid1960s, seven species have been identified as human coronaviruses (HCoVs) (Cui, Li \& Shi 2019).

Basically, coronaviruses are large, positive-sense enveloped ribo nucleic acid (RNA) viruses in the Nidovirales order. They are divided into four genera i.e. $\alpha, \beta, \gamma$ and $\delta$. Three species of $\beta$ coronaviruses have caused outbreaks of deadly pneumonia in humans since the beginning of the 21st century. Human coronaviruses, including hCoV-229E, OC43, NL63, and HKU1, cause mild respiratory diseases. These viruses have a broad distribution among humans and other mammals. Even though most human coronavirus infections are mild, the epidemics of the two betacoronaviruses, severe acute respiratory syndrome coronavirus (SARS-CoV-1), and Middle East respiratory syndrome coronavirus (MERS-CoV), have caused more than 10,000 cumulative cases in the past two decades, with mortality rates of $10 \%$ for SARS-CoV and $37 \%$ for MERSCoV. Causative agent of current outbreak is a novel betacoronavirus, the 2019 novel coronavirus (SARS-CoV-2) and there was no evidence or published reports about this species of coronaviruses before December 2019 (WHO 1, 2020).

\section{Survey Methodology}

A comprehensive PubMed search was performed during December 20, 2019 and April 03, 2020 utilizing the words: Wuhan Virus, COVID-19, SARs coronavirus, ACE2, S-protein, virulence, clinical presentation, epidemiology, genome, treatment, structure, MERs, pathogenesis and/or pathology alone and in combination with other terms. Initially, 300 research papers were 
screened and 74 research papers with information related to the chosen topic and scientific merit were selected for further review and evaluation by three content experts considering quality, reproducibility, credibility and reputation of the journal. Expert in medicine/pharmacogenomics, microbiology/infectious disease and molecular pathology separately reviewed each paper. Mutual agreement on inclusion of information in this review by all three content experts was the threshold of the study.

\section{Findings}

\section{Clinical Presentation}

Most patients who are infected with COVID-19 will have only mild symptoms. Yet due to a high fatality and morbidity rate it is important to identify those at elevated risk. COVID-19 severe illness may be defined as having the endpoints of needing to be ventilated, placed in intensive care units (ICU) or death. Severe disease seems to be correlated linearly with age up to about the age of 65 where the correlation begins to become exponential. (Guan et al., 2020) The incubation period of this disease is about 4-5 days with about $5 \%$ of the population not showing clinical symptoms for as long as 14 day after initial exposure. (Guan et al, 2020). Nearly everyone with the disease under the age of 15 will not develop severe disease and most will not show symptoms. Thus, this age group can serve as carriers who are infected unknown to themselves and to many other therafter (Li Q et al., 2020). The disease can pass from those that show no clinical symptoms. (Zou et al, 2020). Studies indicate that the virus sheds at high levels from the nose in both symptomatic and non-symptomatic patients in a way more like the influenza virus than the SARS virus.(Wu, Zhen, \& Zheng, 2020) The virus also seems to be able to survive on fomite for 72 hours and in the air as an aerosol for 3-4 hours making transmission possible even 
if an infected person has not been around for hours from the air or days from inanimate objects.(van Doremalen et al., 2020)

The most important symptoms in adults are a fever $88 \%$, cough $67.8 \%$, fatigue $38 \%$, difficulty breathing $18.7 \%$, and myalgia. (Guan et al, 2020). It has been shown however that children may show diarrhea as a sign of infection. (Lu X et al, 2020) For those with clinical symptoms the cough is often followed a day or two later by a slight fever with pneumonia appearing perhaps as early as 2-4 days after the initial cough. The blood labs indicate that about 83.7 percent of those infected have lymphocytopenia and 60.7 percent will have C-reactive protein above $10 \mathrm{mg} /$ liter (Guan et al, 2020). Elevated liver enzymes are also relatively common indicating that the COVID - 19 virus has propensity for the liver which may also limit later therapeutic choices. Lymphocytopenia occurs in $87 \%$ of the cases in contrast to SARs where it is at $18 \%$. This may indicate that COVID-19 may be overwhelming the immune system and/or targeting the immune system itself. Chest X-rays are often benign initially or may show signs of interstitial pneumonia with a ground glass appearance. (Holshue et al., 2020). The pneumonia however may progress rapidly into lobar or bronchopneumonia with CT scans similar to what was found with SARS (Franquet, 2009). In terminal cases the chest X-rays may appear completed whited out. There is also evidence that the neuro-invasion by the COVID-19 virus may play a role in respiratory failure. (Li, Bai \& Hashikawa, 2020)

The severity of pneumonia is often scored with the CURB65 score which uses as criteria 1 point for any of the following conditions: Confusion, Urea $>7$ mmoles, Respiratory rate $>30$, diastolic $\mathbf{B P}<60$, and Age $>65$. In the absence of other risk factors, any score equals to 2 or more the patient is generally admitted and a score equals to 3 or more the patient may be considered for intensive care. (Barlow, Nathwani, \& Davey, 2007). 
Interestingly the following factors in addition to age may play a role in morbidity: hypertension, diabetes, hepatitis B infection, COPD, immunodeficiency and cancer (Guan et al. 2020; Fang, Karakiulakis, \& Roth 2020).

\section{Testing}

The ideal specimen for the COVID-19 diagnosis as per Center for Disease Control (CDC) guidelines is a nasopharyngeal swab. Oropharyngeal, nasal mid-turbinate, or nasal swabs are acceptable alternatives if nasopharyngeal swabs are unavailable. Expectorated sputum should be collected from patients with productive cough; induction of sputum is not recommended. A lower respiratory tract aspirate or bronchoalveolar lavage should be collected from patients who are intubated (CDC, 2020). Molecular techniques have been used successfully to identify infectious agents for many years. Unbiased, high-throughput sequencing is a powerful tool for the discovery of pathogens (Palacios et al, 2008; Armstrong et al., 2019).

Considering ss-RNA nature of viral nucleic acid, and due to the fact that this virus was previously not known, the reliable and suggested method other than culture for SARS-CoV-2 RNA is use of reverse-transcription polymerase chain reaction (RT-PCR) (Patel \& Jernigan 2020). Dr. Wang Jianwei and the team at the Chinese Academy of Medical Sciences, Institute of Pathogen Biology, China were the first to sequence the genome of COVID-19 using next generation sequencing (NGS) (Ren et al, 2020). They obtained bronchoalveolar lavage (BAL) fluid samples from the patients, isolated the DNA and RNA, and then sequenced the genetic material. They reported that most of the viral sequences belonged to the CoV family of viruses, which includes the SARS-CoV and the Middle East respiratory syndrome-related (MERS) CoV (Ren et al, 2020). 
These details were readily available before publication, genome sequence was submitted to NCBI library and provided a great help in diagnosis of early cases. The first PCR tests for COVID-19 were developed very rapidly - within two weeks of the disease being identified - and they are now part of the World Health Organization (WHO)'s recommended protocol for dealing with the disease. A positive test for SARS-CoV-2 generally confirms the diagnosis of COVID19, although false-positive tests are possible. If initial testing is negative but the suspicion for COVID-19 remains, the WHO recommends resampling and testing from multiple respiratory tract sites (WHO 2, 2020).

Negative RT-PCR tests on oropharyngeal swabs despite CT findings suggestive of viral pneumonia, have been reported in some patients who ultimately tested positive for SARS-CoV-2 (Xie et al, 2020)

As compared to RT-PCR, serologic tests are considered more rapid and in this kind of outbreak will be able to provide a rapid and in time diagnosis, once largely available, these serological tests (mostly Enzyme Linked Immuno Sorbent Assay or ELISA based) will help the clinicians to identify patients having current or previous infection, asymptomatic patients and patients with negative PCR results. In one study (Guo et al, reported the detection efficiency by IgM - ELISA higher than that of qPCR method after 5.5 days of symptom onset. They mentioned that positive detection rate was significantly increased (98.6\%) when they combined IgM ELISA assay with PCR for each patient compared to a single qPCR test (51.9\%) (Guo, 2020).

Wenling Wang et. al. (2020) reported presence of Virus in specimens collected from multiple sites. According to them respiratory tract samples most often testing positive for the virus. But the live virus was also detected in feces, implying that SARS-CoV-2 may be transmitted by the 
fecal route. They also found a small percentage of blood samples positive for COVID-19 PCR test, suggesting systemic infection.

\section{SARS-COV-2 Virus}

Coronaviruses are a group of viruses that cause a significant percentage of all common colds in human adults and children. Four human coronavirus including 229E, OC43, NL63, and HKU1 are prevalent and typically cause common cold symptoms in immunocompetent individuals. SARS-CoV which causes SARS, has a unique pathogenesis because it causes both upper and lower respiratory tract infections. SARS-CoV-2 is classified as a novel betacoronavirus belonging to the sarbecovirus subgenus of Coronaviridae family. The genome sequence of SARS-CoV-2 is about $89 \%$ identical to bat SARS-like-CoVZXC21 and $82 \%$ identical to human SARS-CoV (Chan et al., 2020). The severity of disease is most often an important indirect factor in a virus's ability to spread. Because coronaviruses have error prone RNA-dependent RNA polymerases (RdRP), mutations and recombination events frequently occur, resulting in quasispecies diversity that is closely associated with adaptive evolution and the capacity to cause disease. Previous studies have shown that SARS-CoV mutated over the 2002-2004 epidemic to better bind to its cellular receptor and replication in human cells, enhancing virulence (Cui, Li \& Shi, 2019).

The genome of the new SARS-CoV-2, as previously mentioned had $89 \%$ nucleotide homology with bat SARS-like-CoVZXC21 and 82\% with that of human SARS-CoV (Chan et al, 2020). For this reason, the new virus was called SARS-CoV-2. Its single-stranded RNA genome contains 29891 nucleotides, encoding for 9860 amino acids. Although its origins are not entirely understood, these genomic analyses suggest that SARS-CoV-2 probably evolved from a strain found in bats. The potential amplifying mammalian host, intermediate between bats and humans, 
is, however, not known. Since the mutation in the original strain could have directly triggered virulence towards humans, it is not certain that this intermediary exists (Cascello et al,. 2020). Zhang et al (2020) have reported pangolins as the possible intermediate host of the virus, but their roles are waiting for further investigation. Coronaviruses use homo-trimers of the spike (S) glycoprotein to promote host attachment and fusion of the viral and cellular membranes for entry. $\mathrm{S}$ is the main antigen present at the viral surface and is the target of neutralizing antibodies during infection. As a result, it is a focus of vaccine design. $\mathrm{S}$ is a class I viral fusion protein synthesized as a single polypeptide chain precursor of approximately 1,300 amino acids (Bosch et al, 2003).

\section{COVID-19 cellular pathogenesis}

For many coronaviruses, $\mathrm{S}$ is processed by host proteases to generate two subunits, designated $\mathrm{S} 1$ and S2, which remain non-covalently bound in the pre-fusion conformation. The N-terminal S1 subunit comprises four $\beta$-rich domains, designated A, B, C and D, with domain A or B acting as receptor-binding domains in different coronaviruses and contributes to stabilization of the prefusion state of the membrane-anchored C-terminal- S2 subunit, that contains the metastable spring-loaded fusion machinery (Walls et al, 2016). During entry, S2 is further proteolytically cleaved at the S2' site, immediately upstream of the fusion peptide (Millet \& Whittaker, 2015). This second cleavage step occurs for all coronaviruses and is believed to activate the protein for membrane fusion, which takes place via irreversible conformational changes (Belouzard, Chu \& Whittaker, 2009).

For all CoVs, S is further cleaved by host proteases at the so-called S2 0 site located immediately upstream of the fusion peptide. This cleavage has been proposed to activate the protein for membrane fusion via extensive irreversible conformational changes (Pallesen et al, 2017; Li et al 
2020). As a result, coronavirus entry into susceptible cells is considered as a complex process that requires the concerted action of receptor- binding and proteolytic processing of the S protein to promote virus-cell fusion. SARS-CoV and several SARS-related coronaviruses (SARSr-CoV) interact directly with angiotensin-converting enzyme 2 (ACE-2) via SB to enter target cells (Wong et al, 2004).

To see how the S protein from SARS-CoV-2 facilitates viral entry into target host cells it has been reported that the S proteins of SARS and SARS-2 or COVID-19 mediate viral entry into a similar spectrum of cell lines as other human coronaviruses. Resembling SARS, SARS-CoV-2 also employs the same host-cell ACE2 as the receptor for cell entry, and the host cell serine protease TMPRSS2 primes the S protein of SARS-CoV-2 for entry (Hoffmann et al, 2020).

As the ACE2 enzyme is integral in the regulation of blood pressure and the renin-angiotensin system, its involvement in SARS-CoV-2 entry into the host cell may suggest possible mechanisms for pathogenesis of the COVID-19 syndrome. The main role of ACE2 is the degradation of Ang II resulting in the formation of angiotensin 1-7 (A (1-7)) which opposes the actions of Ang II. Another well-recognized role of A (1-7) is in stimulation of hematopoiesis and recovery of circulating white blood cells. Other consequences of the virus-receptor interaction may be co-morbidity with patients with high blood pressure or diabetes being treated with ACE-1 inhibitors or angiotensin-receptor antagonists since both drug classes increase the expression of ACE2 (Tikellis \& Thomas, 2012;Rodgers, \& Dizerga 2013; Lei, 2020).

It is also reported that the recombinant receptor binding domain (RBD) protein bound strongly to human ACE2 (hACE2) and bat ACE2 (bACE2) receptors. They also found it to block the entry of SARS-CoV-2 and SARS-CoV into their respective hACE2-expressing cells, suggesting that it 
may serve as a viral attachment inhibitor against SARS-CoV-2 and SARS-CoV infection (Tai et al., 2020).

ACE2 is a type I transmembrane metallo-carboxy-peptidase with homology to ACE, an enzyme long-known to be a key player in the Renin-Angiotensin system (RAS) and a target for the treatment of hypertension (Riordan, 2003). It is mainly expressed in vascular endothelial cells, the renal tubular epithelium, and in Leydig cells in the testes (Ling et al, 2020). That is why this also has been suggested that this novel SARS CoV-2 might affect male fertility. First evidence about such effect reports the alteration of sex-related hormones under COVID-19. They found serum luteinizing hormone ( $\mathrm{LH})$ significantly elevated and testosterone (T) to LH ratio decreased in COVID-19 patients, which infer to the potential hypogonadism (Ling et al, 2020).

ACE2 degrades Angiotensin II to generate Angiotensin 1-7, thereby, negatively regulating RAS (Tikellis \& Thomas, 2012). To show the interaction between ACE2 and viral entry Hoffmann et al (2020) reported that treating Vero-E6 cells, a monkey kidney cell line known to permit SARSCoV replication, with an Anti-ACE2 Antibody blocked the entry of Vesicular stomatitis virus (VSV) pseudotypes expressing the SARS-CoV-2 S protein. Another important step is priming of S protein by the Transmembrane protease, serine 2 TMPRSS2 that is crucial for SARS-CoV infection of target cells and spread throughout the host. Hoffmann et al.,(2020) investigated if SARS-CoV-2 entry is also dependent on S protein priming by TMPRSS 2 and reported that treatment of the Calu-3 human lung cell line with the serine protease inhibitor camostat mesylate partially blocked entry of VSV pseudotypes expressing the SARS-CoV-2 S protein.

Cao et al (2020) mentioned that a previous study showed that residues near lysine 31, and tyrosine 41, 82-84, and 353-357 in human ACE2 were important for the binding of S-protein in coronavirus but they were not able to find any mutation among different ethnic groups of these 
residues. They also reported that there was a lack of natural resistant mutations for coronavirus S-protein binding in populations. Similarly a study by Zhao et al (2020) reported that ACE-2 has been found to be elevated in Asian men, with Caucasian's and Africans having lower level respectively. Taken together, the differences in allele frequencies of ACE2 coding variants among different populations suggested that the diverse genetic basis might affect ACE2 functions among populations (Cao et al, 2020). It is assumed that the higher levels in Asians should also translate into higher levels of ACE-2 in native American populations, as there are evidences that native American tribes began their migration to the New World about 15,000-25,000 years ago from Siberia and are genetically Asian overall and related to tribes in the Altaic region (Pinotti, 2019).

The earliest cases of SARS-CoV-2 or COVID-19 were identified as "pneumonia of unknown etiology", which was defined as an illness of unknown etiology with fever with or without a recorded temperature, radiographic evidence of pneumonia, low or normal leukocyte count or low lymphocyte count during the early stage of disease and no improvement or worsening symptoms after 3-5 days of antimicrobial treatment per standard clinical guidelines(China CDC, 2020).

If we look for similar clinical features between COVID-19 and previously known betacoronavirus infections we will see that most patients presented with fever, dry cough, dyspnea, and bilateral ground-glass opacities on chest CT scans, and these are the features of SARS-CoV-2 infection that are similar to SARS-CoV and MERS-CoV infections. Yet, few patients with SARS-CoV-2 infection showed prominent upper respiratory tract signs and symptoms (e.g., rhinorrhea, sneezing, or sore throat), indicating that the target cells could be 
located in the lower respiratory tract. Additionally, SARS-COV-2 patients unless a child hardly shows the intestinal signs and symptoms (e.g., diarrhea), as compare to MERS-CoV or SARSCoV infection, where about 20-25\% of patients had diarrhea too (Lee et al, 2003; Assiri, AlTawfiq, \& Al-Rabeeah 2013; Chaolin et al 2020).

Additionally, CoVID-19 also needs to be distinguished from other non-viral causative agents that might cause pneumonia-like symptoms and signs for example Mycoplasma, chlamydia, Streptococcus, Haemophilus, and variety of invasive fungi etc. It is also important to consider that patients with viral pneumonia may also test positive to multiple other viruses, and that is why the severity of a combined virus infection with this COVID-19 or SARS-CoV-2 should not be ignored. Comparing the pathology of SARS-CoV-1, MERS and SARS-CoV-2 it is reported that the predominant visceral macroscopic changes in fatal SARS-CoV-1 cases have been edematous lungs with increased gross weights, multiple areas of congestion, enlargement of lymph nodes in the pulmonary hila and the abdominal cavity, diminished spleen size and reduced spleen weights (Nicholls et al, 2003; Ding et al., 2003).

Large numbers of SARS-CoV particles and genomic sequences were also detected within circulating lymphocytes, monocytes, and lymphoid tissues, epithelial cells of the respiratory tract, the intestinal mucosa, the epithelium of renal distal tubules, neurons in the brain, and tissue-resident macrophages residing in different organs (Gu et al., 2005)

Pathophysiology and virulence mechanisms of CoVs, and therefore also of SARS-CoV-2 have links to the function of the non-structural proteins (nsps), and structural proteins. These nsps are able to block the host innate immune response (Lei, Kusov, \& Hilgenfield 2018). With reference to functions of structural proteins, the envelope plays a crucial role as it promotes viral assembly and release. Though, many of these features need more detailed studies. 


\section{Pulmonary Damage Cascade}

The virus enters the respiratory tract usually through air droplets. There are reports of SARSCOV-2 virus associated cases with mild upper respiratory tract symptoms, suggesting the potential for pre- or oligosymptomatic transmission (Wolfel et al., 2020). Once in the respiratory tract the virus eventually enters the alveoli targeting the ACE2 receptor which is predominant on type II pneumocytes. It is the type II pneumocytes that normally produce surfactant which functions to increase pulmonary compliance, prevent atelectasis at the end of expiration and to help recruit collapsed airways and open these up. The type II pneumocytes are destroyed once infected by COVID-19 during the budding out process from these cells thus, reducing surfactant levels. The destroyed type II pneumocytes then recruit macrophages to destroy the dead debris and tissue. In turn the macrophages secrete interleukin 1, interleukin 6 and tumor necrotic factor TNF $\alpha$. Interleukin-1 forms an important part of the inflammatory system causing vasodilation, increased body temperature, increased sensitivity to pain and localized fluid build- up. Interleukin 1 also increases the number of adhesion molecules in endothelium cells enhancing the migration of neutrophils and lymphocytes to the area. Interleukin 6 mediates acute phase proteins to be produced. TNF induces cachexia, fever and cell death of other infected cells. The alveoli begin to become full of fluid and debris which decreases the diffusion of oxygen across the alveoli causing hypoxemia. Additional, bronchial epithelial denudation, loss of cilia, and squamous metaplasia and acute fibrinous with organizing pneumonia in later stages (Bradley \& Bryan, 2009) (Gu et al., 2005). This in turn can permanently damage the lung. As the lung is further damaged the pneumonia can turn to acute respiratory disease, septic shock and multi organ failure. Interestingly, the use of ACE inhibitors and ARB's by those with hypertension 
causes an upregulation of the ACE2 receptor which may partly explain the increase in risks for those with hypertension.

Comparatively we saw for MER-CoV infection the target receptor was dipeptidyl peptidase 4 (DPP4; also known as CD26), (Meyerholz, Lambertz, \& McCray2016). Targets in the lung included pneumocyte, multinucleated epithelial cells, and bronchial submucosal gland cells. Reported pathogenesis of MERS-CoV infection include exudative diffuse alveolar damage with hyaline membranes, pulmonary edema, type II pneumocyte hyperplasia, interstitial pneumonia, and multinucleate syncytial cells. Bronchial submucosal gland necrosis had also been observed (Ng et al 2016; Alsaad et al. 2018). These bronchial lesions comprise the pathologic basis for the respiratory failure and radiologic abnormalities of MERS-CoV infection and a death rate of nearly 45\%.(Walker, 2016)

\section{Treatment}

In the fight against coronavirus, scientists have come up with three strategies for developing new drugs. The first strategy is to test existing broad-spectrum anti-virals, so, Interferons, ribavirin, and cyclophilin inhibitors used to treat coronavirus pneumonia fall into this category. The second strategy is to use existing molecular databases to screen for molecules that may have therapeutic effect on coronavirus and the third strategy is directly based on the genomic information and pathological characteristics of different coronaviruses to develop new targeted drugs from scratch (Wu et al., 2020)

Among some of the treatments that are currently on hand and have been used in China and other places are zinc, chloroquine/hydroxychloroquine, and Azithromycin. Though each of these inexpensive medications have not yet been approved by the FDA for the treatment of COVID-19 
virus they have been approved for other disorders and have been used extensively overseas in the treatment of the COVID-19 virus. (Gao et al., 2020)

Zinc

Zinc has been shown to limit the duration of colds (many of which are caused by coronaviruses) by $40 \%$ in duration. (Hemila, 2017) Zinc is the most common trace metal found in the human cell following iron. Zinc is a cofactor for many enzymes that help maintain a healthy immune system and is involved in nearly every aspect of immune response. Zinc is especially important for humoral immunity that is the release of antibodies that opsonize the COVID-19 virus for phagocytosis by immune cells, kill infected cells by the NK cells and block the entrance of the virus into the cells. (Skrajnowska \& Bobrowska-Korczak, 2019). Zinc also may also interfere with RNA dependent RNA polymerase which is essential for the replication of the COVID-19 virus (Kaushik et al. 2017). Zinc enters the cell through the Zrt1p and Zrt2p transporters and can also utilize the voltage-dependent calcium channels, NMDA receptors and AMPA-Rs receptors. (Inoue, Bryant, \& Xiong, 2015)

\section{Chloroquine/Hydroxychloroquine}

Chloroquine is a very inexpensive drug and has been around since 1934 having been used as an antimalarial and immunosuppressant. Chloroquine has been shown to form an ionophore in the cellular membrane allowing doubling the amount of zinc that can enter the cell at clinically relevant levels(Xue et al, 2020) Chloroquine is a very alkaline medication that can readily distribute throughout one's cells and settle in those areas that are especially acidic such as the endosomes which are small vacuoles that the COVID -19 virus uses to enter the cell after attachment at the cellular membrane. Chloroquine can increase the $\mathrm{pH}$ of the endosome 
inhibiting the ability of the COVID-19 virus to enter the cell. (Wang et al. 2020), In a clinical trial of 100 patients in China using chloroquine it was found that the course of the disease was shortened, and the pneumonia exasperation inhibited. (Gao, Tian, \& Yang 2020). No serious adverse effects were seen. Hydroxychloroquine may have greater inhibitory potential than chloroquine and may be safer as it does not have the same risk to cause retinal damage.

\section{Azithromycin}

Azithromycin may serve a dual purpose in the treatment of COVID-19 by reducing the risk of secondary bacterial infections and by taking advantage of recently uncovered anti-viral properties. As an anti-viral is has been shown in patients with compromised lung function to induce interferon- $\beta$ and interferon- $\gamma$. (Schogler et al., 2015; Geilin. Johnston, \& Edwards 2015). Interferons are critical for innate and adaptive immunity against viral infections. Additionally, Azithromycin inhibits the bronchial excretion of mucin which when excreted narrows the bronchial passageways inhibiting breathing.Azithromycin may also limit inflammation. (Min \& Jang, 2012).

lopinavir-ritonavir

There are several COVID-19 clinical trials looking at different combinations of Interferons with and without antivirals. A systematic review of the evidence related to lopinavir-ritonavir and SARS and MERS suggests it could be a potential treatment for COVID-19 infections. Lopinavir/Ritonavir with or without interferons are being used regularly in the treatment of COVID-19 (Yao et al., 2020).

\section{Summary/Conclusion}


The COVID-19 pandemic is the third coronavirus-caused disease to arise in the $21^{\text {st }}$ century. Cultural and lifestyle changes of the last fifty years have created a world in which these newly seen viral infections can spread worldwide in day and weeks. The need to prepare for future pandemics should be obvious.

Rapid identification, basic public health interventions such as social distancing, followed as quickly as possible by development of diagnostic tests to monitor in real time the spread of the disease should become standard reactions to new viral disease. Preparatory measure such as availability of enough personal protective equipments, medication, as well as costly instrumentation (such as ventilators) is essential to overcome the situations like current pandemic. SARS-CoV-2 mutates rapidly, infects with high efficiency, and causes severe illness in a high proportion of infections (Liu Y, et al., 2020). These qualities should be expected in future outbreaks. Entry of the virus via interaction with ACE-2 suggest a variety of potential therapeutic directions to develop and some effort should be made to develop pan-coronavirus interventions. ACE-2 shows patterns of ethnic and anatomic expression consistent with the spread of the disease among ethnic groups and the pulmonary and immune suppression that are major features of the severe acute respiratory syndrome -Coronavirus 2 (SARS-COV-2 syndrome). Public health CANDOR regarding future infections; will prepare the public for realistic scenarios and greater compliance with traumatic interventions ranging from social distancing, stay-at-home orders, job loss, economic costs and end-of-life care that will enhance our ability to conquer future pandemics faster and at less cost than it will require to overcome COVID-19.

\section{Acknowledgements}


Bonnie Grunther for reviewing the manuscript for consistency, grammar, use of language and syntax.

\section{References}

Assiri A, Al-Tawfiq JA, \& Al-Rabeeah AA (2013). Epidemiological , demographic and clinicalcharacteristics of 47 cases of Middle East Respiratory Syndrome Coronavirus Disease from Saudi Arabia: A Descriptive Study. Lancet Inf Dis 13:752-61.

Armstrong GL, MacCannell DR, Taylor J, et a1. (2019). Pathogen genomics in public health. N Engl J Med 381:2569-2580.

Alsaad KO, Hajeer AH, Al Balwi M, et al. (2018). Histopathology of Middle East respiratory syndrome coronovirus (MERS-CoV) infection - clinicopathological and ultrastructural study. Histopathology. 72(3):516-524.

Barlow G1, Nathwani D, \& Davey P (2007). The CURB65 pneumonia severity score outperforms generic sepsis and early warning scores in predicting mortality in communityacquired pneumonia. Thorax Mar;62:253-9.

Bosch BJ, van der Zee R, de Haan CA, \& Rottier PJ (2003). The coronavirus spike protein is a class I virus fusion protein: structural and functional characterization of the fusion core complex. J. Virol. 77:8801-8811.

Belouzard S, Chu VC, \& Whittaker GR (2009). Activation of the SARS coronavirus spike protein via sequential proteolytic cleavage at two distinct sites. Proc. Natl Acad. Sci. USA. 106:5871-5876. 
Bradley BT, Bryan A (2019). Emerging respiratory infections: The infectious disease pathology of SARS, MERS, pandemic influenza, and Legionella. Semin Diagn Pathol. 36(3):152-159

Cui J, Li F, \& Shi ZL (2019). Origin and evolution of pathogenic coronaviruses. Nat Rev Microbiol 17:181e92.

Chan JF-W, Kok K-H, Zhu Z, et al. (2020). Genomic characterization of the 2019 novel human-pathogenic coronavirus isolated from patients with acute respiratory disease in Wuhan, Hubei, China. Emerg Microb Infect. https://doi.org/10.1080/22221751.2020.1719902 [in press]

Cao Y, Lin I, Feng Z, et al. (2020). Comparative Genetic Analysis of the Novel Coronavirus (2019-nCoV/SARS-CoV-2) Receptor ACE2 in Different Populations. Cell Discovery. 6:11

Cascello M. (2020). Features, Evaluation and Treatment Coronavirus (COVID-19). https://www.ncbi.nlm.nih.gov/books/NBK554776/.

Centers for Disease Control and Prevention (2020). Interim Guidelines for Collecting, Handling, and Testing Clinical Specimens from Persons under Investigation (PUIs) for Coronavirus Disease 2019 COVID-19 (2020). https://www.cdc.gov/coronavirus/2019nCoV/lab/guidelines-clinical-specimens.html (Accessed on March 15, 2020).

Chaolin Huang, Yeming Wang, et al. (2020). Clinical features of patients infected with 2019 novel coronavirus in Wuhan, China. Lancet 395: 497-506. doi.org/10.1016/S01406736(20)30183-5.

China CDC (2020), An outbreak of NCIP (2019-nCov) infection in China- Wuhan HUubei province , 2019-2020.China CDC Weekly 2(5):79-80. 
Coutard B, Valle C., de Lamballerie X, et al (2020). The Spike Glycoprotein of the New Coronavirus 2019-nCoV Contains a Furin-like Cleavage Site Absent in CoV of the Same Clade. Antiviral Research https://doi.org/10.1016/j.antiviral.2020.104742.

Cui J, Li F, \& Shi ZL (2019), Origin and evolution of pathogenic coronaviruses. Nat Rev Microbiol. DOI: 0.1038/s41579-018-0118-9.

COVID Data: Coronavirus COVID-19 Global Cases by the Center for Systems Science and Engineering, Johns Hopkins University. Accessed Mar 31, 2020. https://gisanddata.maps.arcgis.com/apps/opsdashboard/index.html\#/bda7594740fd40299423467 b48e9ecf6

Ding Y, Wang H, Shen H, et al (2003). The clinical pathology of severe acute respiratory syndrome (SARS): a report from China. J Pathol. 200(3):282-289

Fang L, Karakiulakis G, Roth M (2020). Are patients with hypertension and diabetes mellitus at increased risk for COVID-19 infection? The Lancet, DOI: 10.1016/S2213-2600(20)30116-8.

Franquet T (2009). Imaging of Pulmonary Viral Pneumonia. Radiology ;260:18-39.

Gao J, Tian Z, \& Yang X (2020). Breakthrough: Chloroquine Phosphate has Shown Apparent Efficacy in Treatment of COVID-19 Associated Pneumonia in Clinical Studies. BioScience Trends. 14(1)72-3.

Gielen V, Johnston S.L., \& Edwards MR (2010). Azithromycin induces anti-viral responses in bronchial epithelial cells. Eur Respir J 36:646-54.

Guan W, Ni Z, Hu Y, et al. (2020). Clinical Characteristics of Coronavirus Disease 2019 in China. N Engl J Med. DOI:10.1056/NEJMoa2002032. 
Guo L, Ren L, Yang S, et al. (2020). Profiling Early Humoral Response to Diagnose Novel Coronavirus Disease (COVID-19). Clin Infect Dis. DOI: 10.1093/cid/ciaa310.

Gu J, Gong E, Zhang B, et al. (2005). Multiple organ infection and the pathogenesis of SARS. J Exp Med. 202(3):415-424. http://wjw.wuhan.gov.cn/front/web/showDetail/2019123108989. Report of clustering pneumonia of unknown etiology in Wuhan City. 2019.

Gao J et al. (2020). Breakthrough: Chloroquine phosphate has shown apparent efficacy in treatment of COVID-19 associated pneumonia in clinical studies. BioScience Trends. 2020; 14(1):72-73. DOI: $10.5582 /$ bst.2020.01047

Hagen A (2020). ASM COVID-19 International Summit Sets Research Priorities, Amer Society for Microbiology.https://asm.org/Articles/2020/March/ASM-COVID-19-International-SummitSets-Research Pr?utm_campaign=Articles\&utm_id=00411cw2e8jc714\&utm_medium=social\&utm_source=fac ebook.

Hu LL, Wang WJ, Zhu QJ, Yang L(2020). [Novel coronavirus pneumonia related liver injury: etiological analysis and treatment strategy]. Zhonghua Gan Zang Bing Za Zhi. 28:E001.

Hemila H (2017). Zinc lozenges and the common cold: a meta-analysis comparing zinc acetate and zinc gluconate, and the role of zinc dosage. JRSM DOI: 10.1177/2054270417694291

Holshue ML, Debolt C, Lindquist S, et al. (2020). First Case of 2019 Novel Coronavirus in the United States. N Engl J Med, DOI:10.1056/NEJMoa2001191. 
Hoffmann M, Kleine-Weber H, Schroeder S. et al.(2020). SARS-CoV-2 cell entry depends on ACE2 and TMPRSS2 and is blocked by a clinically proven protease inhibitor. Cell Mar 5; [epub]. https://doi.org/10.1016/j.cell.2020.02.052.

Heneghan Carl et al. (2020). SARS-CoV-2 viral load and the severity of COVID-19.CEBM. https://www.cebm.net/covid-19/sars-cov-2-viral-load-and-the-severity-of-covid-19/

Inoue K, O’Bryant Z,\& Xiong ZG, (2015). Zinc-Permeable Ion Channels: Effects on Intracellular Zinc Dynamics and Potential Physiological/Pathophysiological Significance.Curr Med Chem 22:1248-57.

Kaushik N, Subramani C, Anang S, et al. (2017). Zinc Salts Block Hepatitis E Virus Replication by Inhibiting the Activity of Viral RNA-Dependent RNA Polymerase. Journal of Virology 91;1-13.

Jiang, F. Yang J, Zhang Y, et al (2015). Angiotensin-converting enzyme 2 and angiotensin 1-7: novel therapeutic targets. Nat. Rev. Cardiol. 11:413.

Li Q, Guan X, Wu P, et al.(2020). Early Transmission Dynamics in Wuhan, China of Novel Coronavirus-infected Pneumonia. N Engl J Med. DOI:10.1056/NEJMoa200136.

Li YC, Bai WZ, \& Hashikawa T,(2020). The Neuroinvasive Potential of SARS-CoV2 May a Role in the Respiratory Failure of COVID-19 Patients. J Med Vir. DOI: 10.1002/jmv.25728.

Liu Y, Gayle AA, Wilder-Smith A, \& Rocklov J (2020). The Reproductive Number of COVID-19 is Higher Compared to SARS Coronavirus. Journal of Travel Medicine. DOI: 10.1093/jtmtaaa021. 
Kuba, K. mai Y, Ohto-Nakanishi T, Penninger JM. et al. (2010). Trilogy of ACE2: A peptidase in the renin-angiotensin system, a SARS receptor, and a partner for amino acid transporters. Pharmacol. Ther. 128:119.

Lu X, Zang Y, Li Y, et al. (2020). SAR-CoV-2 Infection in Children, N Engl J Med. DOI:10.1056/NEJMc2005073.

Ling Ma, Xi W, Li D et al.(2020). Effect of SARS-CoV-2 infection upon male gonadal function: A single center-based study.MEDRXiv

doi: https://doi.org/10.1101/2020.03.21.20037267

Lee N Hui D Wu A et al. (2003). A major outbreak of severe acute respiratory syndrome in Hong Kong. N Engl J Med. 348: 1986-1994

Lei J, Kusov Y, Hilgenfeld R. (2018). Nsp3 of coronaviruses: Structures and functions of a large multi-domain protein. Antiviral Res. Jan;149:58-74

Min JY, Jang YJ. (2012). Macrolide Therapy in Respiratory Viral Infections. Mediators Inflamm. DOI: 10.1155/2012/649570.

Meyerholz DK, Lambertz AM, McCray PB Jr. (2016). Dipeptidyl peptidase 4 distribution in the human respiratory tract: implications for the Middle East respiratory syndrome. Am J Pathol. 186(1):78-86.

Madjid M, Safavi-Naeini P, Solomon SD, Vardeny O. (2020). Potential effects of coronaviruses on the cardiovascular system: a review. JAMA Cardiol. Published online doi:10.1001/jamacardio.2020.1286 
Ng DL, Al Hosani F, Keating MK, et al. (2014). Clinicopathologic, immunohistochemical, and ultrastructural findings of a fatal case of Middle East respiratory syndrome coronavirus infection in the United Arab Emirates, Am J Pathol.186(3):652-658.

Nicholls JM, Poon LL, Lee KC, et al. (2003). Lung pathology of fatal severe acute respiratory syndrome. Lancet. 361(9371):1773-1778.

Palacios G, Druce J, Du L, et al. (2008) A new arenavirus in a cluster of fatal transplantassociated diseases. N Engl J Med 358:991-998.

Pinotti T, Bergström A, Geppert M, et al. (2019). Y Chromosome Sequences Reveal a Short Beringian Standstill, Rapid Expansion, and early Population structure of Native American Founders. Curr Bio DOI: 10.1016/j.cub.2018.11.029.

Patel A, \& Jernigan DB, (2020). 2019-nCoV CDC Response Team. Initial Public Health Response and Interim Clinical Guidance for the 2019 Novel Coronavirus Outbreak - United States, December 31, 2019-February 4, 2020. MMWR Morb Mortal Wkly Rep 69:140.

Pallesen, J Wang N, Corbett KS. et al. (2017). Immunogenicity and structures of a rationally designed prefusion MERS-CoV spike antigen. Proc. Natl Acad. Sci. USA 114, E7348-E7357

Rothe C, Schunk M, Sothman M, et al. (2020). Transmission of 2019-nCoV Infection from an Asymptomatic Contact in Germany. N Engl J Med DOI:10.1056/NEJMc2001468.

Ren LL, Wang YM, Wu ZQ et al. (2020) Identification of a novel coronavirus causing severe pneumonia in human. Chinese Medical Journal DOI: 10.1097/CM9.0000000000000722.

Riordan, J.F (2003). Angiotensin-I-converting enzyme and its relatives. Genome Bio. 4:225. 
Rodgers KE, Dizerega GS(2013).Contribution of the Local RAS to Hematopoietic Function: A Novel Therapeutic Target.Front Endocrinol (Lausanne). Oct 23; 4:157.

Schogler A, Kopf B, Edwards MR, et al. (2015). Novel Antiviral Properties of Azithromycin in Cystic Fibrosis Airway Epithelial Cells. Cystic Fibrosis. DOI: 10.1183/09031936.00102014.

Skrajnowska D, Bobrowska-Korczak B (2019). Role of Zinc in Immune System and AntiCancer Defense Mechanisms. Nutrients ;11: 2273.

Sodhi C, Wohlford-Lenane C,Yamaguchi Y, et al.(2018). Attenuation of Pulmonary ACE2 Activity Impairs Inactivation of des-Arg ${ }^{9}$ bradykinin/BKB1R Axis and Facilitates LPS-induced Neutrophil Infiltration. Am J Physiol Lung Cell Mol Physiol :314:L17-L31.

Tai, W., He, L., Zhang, X. et al.(2020). Characterization of the receptor-binding domain (RBD) of 2019 novel coronavirus: implication for development of RBD protein as a viral attachment inhibitor and vaccine. Cell Mol Immunol. https://doi.org/10.1038/s41423-020-0400-4.

Tikellis, C and M.C. Thomas (2012). Angiotensin-Converting Enzyme 2 (ACE2) Is a Key Modulator of the Renin Angiotensin System in Health and Disease. Intl. J. Peptides DOI: $10.1155 / 2012 / 256294$

Vankadari N and Wilce JA. Emerging WuHan (2020). (COVID-19) coronavirus: glycan shield and structure prediction of spike glycoprotein and its interaction with human CD26. Emerging Microbes \& Infections. 9:601-4.

Van Doremalen N, Bushmaker T, Morris D, et al.(2020). Aerosol and Surface Stability of SAR-CoV-2 as Compared with SARS-CoV-1. N Engl J Med. DOI:10.1056/NEJMc2004973. 
Wang M, Cao R, Zhang L, et al. (2020). Remdesivir and Chloroquine Effectively Inhibit the Recently Emerged Novel Coronavirus (2019-nCoV) in Vitro. Cell Research. 30:269-271.

Wang W, Xu Y, Gao R et al.(2020). Detection of SARS-CoV-2 in Different Types of Clinical Specimens. JAMA. Published online 2020. doi:10.1001/jama.2020.3786.

Walls AC (2016). Cryo-electron microscopy structure of a coronavirus spike glycoprotein trimer. Nature. 2016;531:114-117.

Walker DH (2016). Value of autopsy emphasized in the case report of a single patient with Middle East respiratory syndrome. Am J Pathol. 186(3):507-510

Wong, S. K., Li, W., Moore, M. J., Choe, H. \& Farzan, M.(2004) A 193-amino acid fragment of the SARS coronavirus S protein efficiently binds angiotensin-converting enzyme 2 . The Journal of biological chemistry 279, 3197-3201, https://doi.org/10.1074/jbc.C300520200.

World Health Organization (2003), Summary of probable SARS cases with onset of illness from 1 November 2002 to 31 July 2003.

https://www.who.int/csr/sars/country/table2004_04_21/en/. (Accessed Mar 22, 2020)

World Health Organization (2020). Coronavirus disease (COVID-19) technical guidance:

Surveillance and case definitions. https://www.who.int/emergencies/diseases/novel-coronavirus2019/technical-guidance/surveillance-and-case-definitions (Accessed on February 28, 2020).

Wu C, Zheng S, Chen Y, Zheng M.(2020). Single - cell RNA Expression Profiling of ACE2, the Putative Receptor of Wuhan 2019-nCoV, in the Nasal Tissue.BioRXiv DOI:

$10.1101 / 2020.02 .11 .20022228$ 
Wu C et al. (2020). Analysis of therapeutic targets for SARS-CoV-2 and discovery of potential drugs by computational methods, Acta Pharmaceutica Sinica B, https://doi.org/10.1016/j.apsb.2020.02.008.

Wolfel R et al. (2020). Virological assessment of hospitalized patients with COVID-2019 Nature (2020). https://doi.org/10.1038/s41586-020-2196-X

Xue J, Moyer A, Peng B, et al. (2020). Chloroquine Is a Zinc Ionophore, PLOS one DOI:10.1371/journal.pone.0109180.

Xie X, Zhong Z, Zhao W et al. (2020). Chest CT for Typical 2019-nCoV Pneumonia:

Relationship to Negative RT-PCR Testing. Radiology. 12:200343. doi:

10.1148/radiol.2020200343. [Epub ahead of print]

Yao N, Wang SN, Lian JQ, et al. (2020). Clinical characteristics and influencing factors of patients with novel coronavirus pneumonia combined with liver injury in Shaanxi region. Zhonghua Gan Zang Bing Za Zhi. 28:E003.

Yao TT et al (2020) A Systematic Review of Lopinavir Therapy for SARS Coronavirus and MERS Coronavirus-A Possible Reference for Coronavirus Disease-19 Treatment Option. J Med Virol. 2020 Feb 27. doi: 10.1002/jmv.25729. [Epub ahead of print] Available at: https://www.ncbi.nlm.nih.gov/pubmed/32104907 (accessed 4.3.2020)

Yao X, Ye F, Zhang M, et al. (2020). In Vitro Antiviral Activity and Projection of Optimizing Dosing Design of Hydroxychloroquine for the Treatment of Severe Acute Respiratory Syndrome Coronavirus-2(SARS-CoV-2). Clin Infect Dis. DOI: 10.1093/cid/ciaa237. 
Yuan, Y. Cao D, Zhang Y et al. (2017). Cryo-EM structures of MERS-CoV and SARS-CoV spike glycoproteins reveal the dynamic receptor binding domains. Nat. Commun. 8, 15092

Zhao D, Yao F, Wang, et al. (2020). A Comparative Study on the Clinical Features of COVID19 Pneumonia to Other Pneumonias. Clin Inf Dis. DOI: 10.1093/cid/ciaa247.

Zhao Y, Zhao Z, Wang Y, et al. (2020). Single-cell RNA Expression of Profiling of ACE2, the Putative Receptor of Wuhan 2019-nCov. BioRxiv doi:

https://doi.org/10.1101/2020.01.26.919985.

Zou L, Ruan F, Huang M, et al. (2020). SARS-COV-2 Viral Load in Upper Respiratory Specimens of Infected Patients. N Engl J Med. DOI:10.1056/NEJMc2001737.

Zheng J. (2020). SARS-CoV-2: an Emerging Coronavirus that Causes a Global Threat. International journal of biological sciences, 16(10), 1678-1685. https://doi.org/10.7150/ijbs.45053 